\title{
Role of cytokine release during lung resection surgery and postoperatory complications
}

\section{Casanova Barea J., González M F., De la Gala F., Garutti I., Vera Sanchez M.B., Sastre I. Anesthesiology Department. Gregorio Maranon Hospital. Madrid.}

Background and Goal of Study: During lung resection surgery (LRS) with periods of one-lung ventilation (OLV), inflammatory response is multifactorial. Several authors have demonstrated that perioperative exaggerated inflammatory response could lead to postoperative surgical complications. In the last decade, has been proposed the Clavien-Dindo classification to evaluate the impact of postoperative complications on postoperative outcome. The aim of the study was to analyse the impact of intra and early postoperative local and systemic inflammatory response on the incidence of postoperative complications after LRS evaluated by Clavien-Dindo classification.

Materials and Methods: Follow-up of 174 patients undergoing pulmonary resection surgery. 84 segmentectomies, 79 lobectomies and 11 pneumonectomies or bilobectomies. Patients were divided into three groups according to Clavien Dindo classification. A first group ( 0 ) in which there were no complications. A second group (1 and 2 ) encompassing grades I and II of Clavien Dindo and a third group (3,4 and 5) encompassing classes III, IV and V. Blood samples were obtained for biochemical analysis of inflammatory response at the following moments: after anesthetic induction (baseline), at 30 minutes after initiation of OLV (OLV30), at the end of OLV (OLV END)and at 6 (6H PO) and 18 (18H PO) postoperative hours. Bronchoalveolar lavage was performed after induction and at the end of OLV in both lung operated and ventilated lung for biochemical analysis.

IL 6 BLOOD

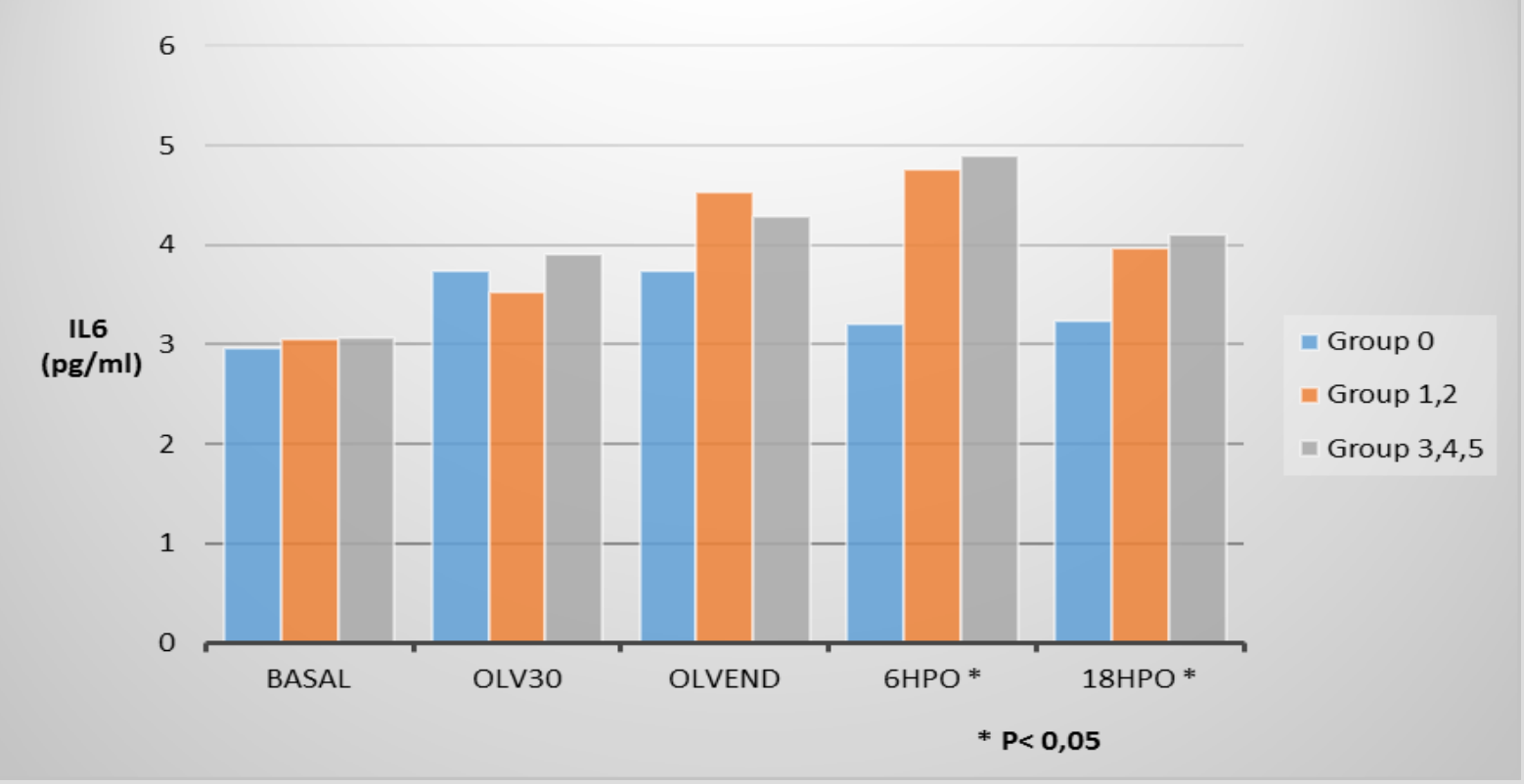

Figure 1

IL 10 BRONCHOALVEOLAR LAVAGE

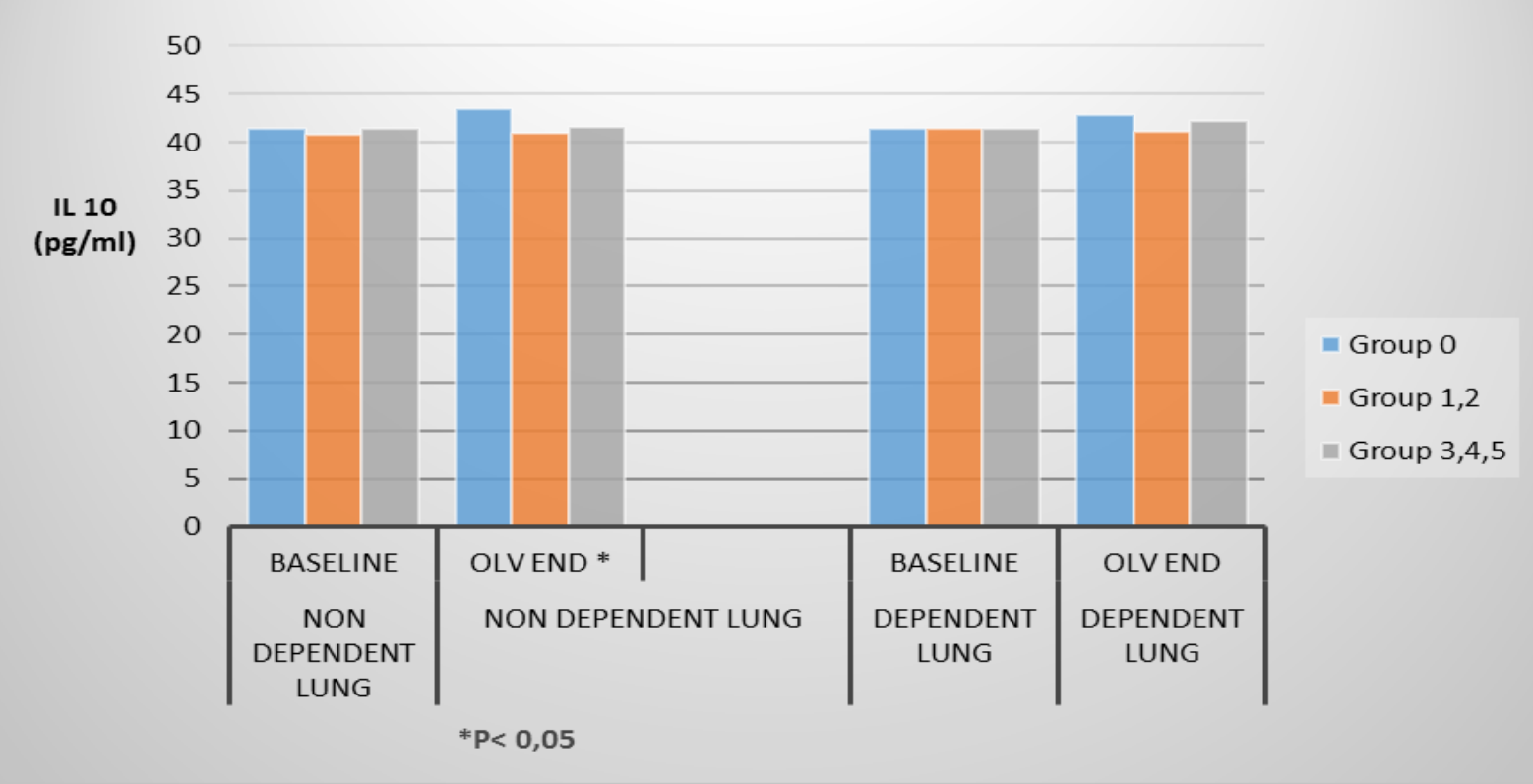

Figure 3

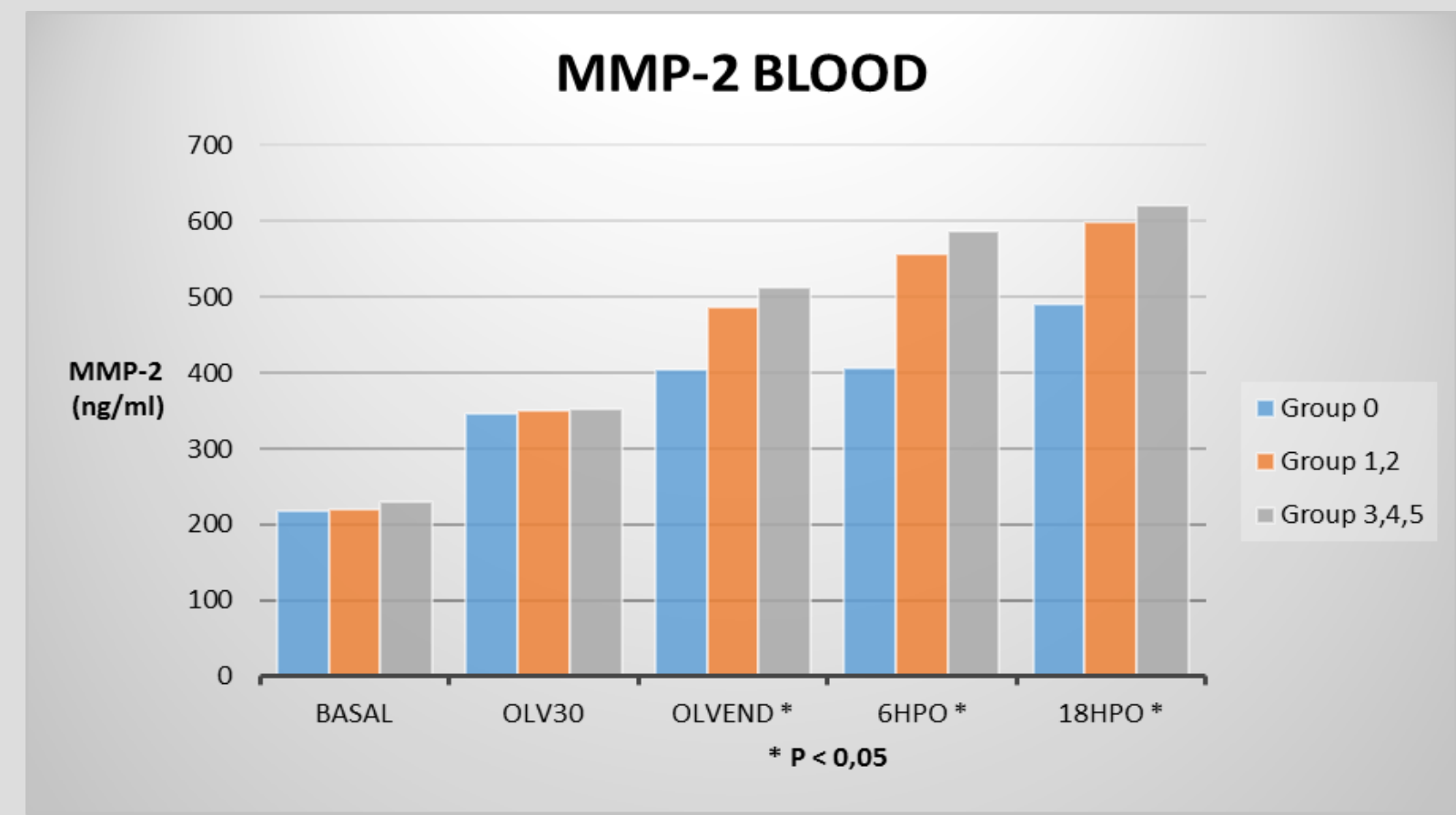

Figure 2

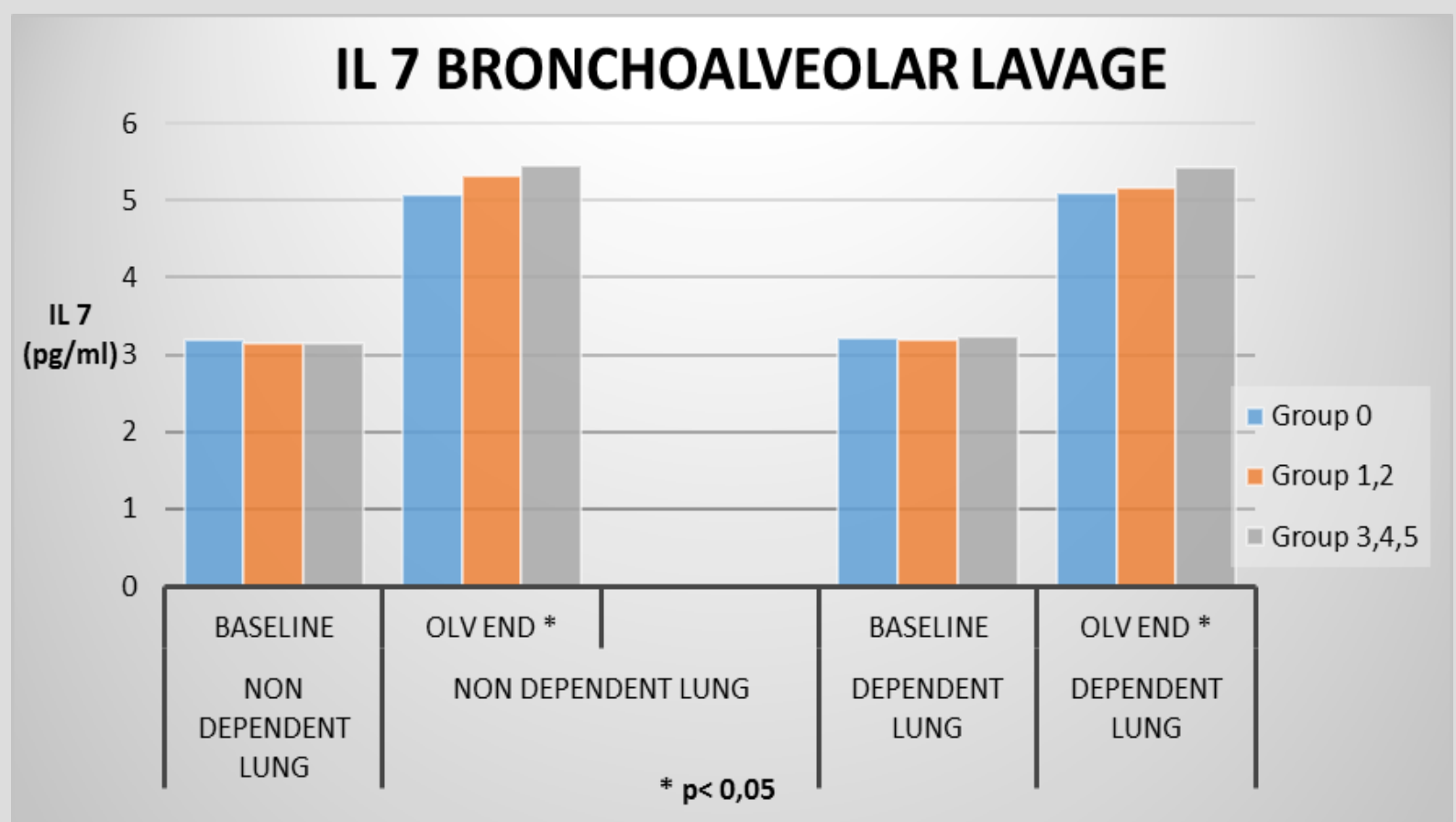

Figure 4

Results and discussion: Figures 1 to 4 , show the more relevant results. Patients with less perioperative inflammatory response (lower IL 6, MMP 2, IL 7 levels and higher IL 10 levels) showed better postoperative outcome. In major surgery we recommend taking inflammation biomarkers measurements, to detect high-risk patients in developing postoperative complications. It'll allow the early instauration of clinical measures to improve the outcome. Conclusion: Proinflammatory cytokines release and anti-inflammatory decrease, determine the appearance of postoperative complications and their severity.

\section{References:}

1 - Dindo D, et al. Ann Surg. 2004. 